\title{
The photosphere and circumstellar environment of the Be star Achernar
}

\author{
Daniel M. Faes ${ }^{1,2}$, Armando Domiciano de Souza ${ }^{2}$, Alex C. Carciofi ${ }^{1}$ \\ and Philippe Bendjoya ${ }^{2}$ \\ ${ }^{1}$ Instituto de Astronomia, Geofísica e Ciências Atmosféricas, Universidade de São Paulo, Rua \\ do Matão 1226, Cidade Universitária, 05508-900, São Paulo, SP, Brazil \\ email: moser@usp.br \\ ${ }^{2}$ Lab. J.-L. Lagrange, UMR 7293 - Observatoire de la Côte d'Azur (OCA), Univ. de \\ Nice-Sophia Antipolis (UNS), CNRS, Valrose, 06108 Nice, France
}

\begin{abstract}
Achernar is a key target to investigate high stellar rotation and the Be phenonemon. It is also the hottest star for which detailed photospheric information is available. Here we report our results to determine the photospheric parameters of Achernar and evaluate how the emission of a Viscous Decretion Disk (VDD) around it would be observable. The analysis is based on interferometric data (PIONIER and AMBER at ESO-VLTI), complemented by spectroscopy and polarimetry for the circumstellar emission. For the first time fundamental parameters of a Be photosphere were determined. The presence of a residual disk at the quiescent phase and some characteristics of the new formed disk (2013 activity) are also discussed. This is rare opportunity to precisely determine the stellar brightness distribution and evaluate the evolution of a just formed Be disk.
\end{abstract}

Keywords. stars: individual (Achernar), stars: fundamental parameters, techniques: interferometric, circumstellar matter, stars: emission-line, Be

\section{Introduction}

Be stars are known to be rapid rotators and this property is believed to be fundamentally linked to the existence of the disk. Other mechanisms, such as magnetic activity, non-radial pulsations and stochastic processes at the photosphere are also necessary agents to explain the Be phenomenon. The investigation of the photosphere of Be stars, and of their circumstellar (CS) disks, has entered a new phase during last years, both observationally and theoretically. See Rivinius et al. (2013b) for a review.

Optical and infrared interferometry is a technique capable of bringing qualitatively new information by resolving the stars and their disks at the milliarcsecond (mas) level. Due to the relatively strict magnitude limits of current interferometers, bright nearby Be stars are among the most popular and most frequently observed targets.

\section{An interferometric review of Achernar}

Achernar ( $\alpha$ Eridani, HD10144) is the closest and brightest Be star in the sky. It is also the hottest and more massive star from which we have detailed photospheric information (van Belle 2012). Moreover, among them, it is the one of the highest rotation rate (Domiciano de Souza et al. 2014).

The successive generations of beam combiners of ESO-VLTI (Very Large Telescope Interferometer) have being used to study Achernar. This section summarize these early interferometric studies. All parameters derived from interferometry strongly depend on 
modeling (i.e., accurate brightness distribution), which is wavelength dependent. Nevertheless, fundamental physical properties from both photosphere and CS environment could be constrained based on the high-angular resolution information available.

\subsection{First oblateness determination with VINCI}

Domiciano de Souza et al. (2003) determined for the first time the oblateness of Achernar (and of a Be star) with $K$-band visibility data from VINCI. It was also the first estimation of the on-sky orientation of the star. The diameter ratio between the equator and the pole $2 a / 2 b=1.56$ was found adjusting to each projected position angle (PA) of the observations the size of an uniform disc.

Although undoubtedly indicating a high rotation rate for the star, that was not necessarily implying a rotation above the Roche limit: the radii ratio depends on the underlying stellar model (the fit of multiple uniform disks is just a first approximation of the stellar brightness distribution). Knowing that the star had a little activity at the time of the observations (e.g. Vinicius et al. 2006), the authors have also explicitly ignored the contribution of the CS component in the data since it was a much weaker emission than the stellar one $(<5 \%)$.

\subsection{On a polar emission in the interferometric signal}

Kervella \& Domiciano de Souza (2006) analyzed Achernar's VINCI data from both $K$ and $H$ bands. They have done a single fit on them adopting a uniform ellipse brightness distribution for the star. The diameter ratio between the equator and the pole was then $2 a / 2 b=1.41$.

In this scenario, the addition of a elongated CS envelope with Gaussian brightness distribution superimposed to the stellar model significantly improved the quality of the fitting. This CS component had $\approx 4.5 \%$ of the total flux and removed the trend that appeared in the residuals of the uniform ellipse in PAs around the polar direction. This study was made using only the VINCI data as constraint, and did not relate the CS emission with other observables.

\subsection{The first multi-technique analyzes}

Carciofi et al. (2008) and Kanaan et al. (2008) have independently done a multi-technique fitting of Achernar at the time of VINCI observations. They have used SED and $\mathrm{H} \alpha$ line profiles as constraints, and linked them with polarimetric estimates. Although taking into account the brightness distribution by the Von Zeipel effect and the Roche geometry due to the high rotation of the star, they found distinct results: while Carciofi et al. argue that the presence of a residual CS disk in Achernar is sufficient to explain the observed quantities with a near-critical rotating star, Kanaan et al. conclude that at the time of the VINCI observations Achernar had either a small or no CS disk, but it did have a polar, stellar wind.

\subsection{Proof of concept: what information is contained on diff. phases}

Domiciano de Souza et al. (2012a) have done the first consistent determination of multiple photospheric parameters of Achernar (radius, rotational velocity, inclination angle and on-sky orientation). This work was based on AMBER high resolution spectrointerferometry along the HBrackett $\gamma$ line. The photospheric parameters were found using the CHARRON code (Domiciano de Souza et al. 2002, 2012b) fitting the differential phases. The derived equatorial angular diameter was compatible with previous values from visibilities, and the diameter ratio was below the Roche limit $(2 a / 2 b=1.45)$. This 
Table 1. Physical parameters of Achernar derived from the fit of the RVZ model (CHARRON code) to VLTI/PIONIER H band data using the MCMC method (emcee code).

\begin{tabular}{|c|c|}
\hline Model free parameters fitted & Values and uncertainties \\
\hline Equatorial radius $\left(R_{\odot}\right)$ & $9.16(+0.23 ;-0.23)$ \\
Equatorial rotation velocity $\left(\mathrm{km} \mathrm{s}^{-1}\right)$ & $298.8(+6.9 ;-5.5)$ \\
Rotation-axis inclination angle $\left(^{\circ}\right)$ & $60.6(+7.1 ;-3.9)$ \\
Gravity-darkening coefficient $(\beta)$ & $0.166(+0.012 ;-0.010)$ \\
Position angle of the visible pole $\left(^{\circ}\right)$ & $216.9(+0.4 ;-0.4)$ \\
\hline
\end{tabular}

time, no positive detection of circumstellar activity was found at the time of observations (2009-2010), from both polarimetry and spectroscopy.

This study shows that differential phases are capable of providing useful information, but multiple parameter fitting using only a differential quantity is tricky (see Domiciano de Souza et al. 2014, for a discussion).

\section{The photospheric characterization with PIONIER}

A quantitative jump was given to the characterization of Achernar by Domiciano de Souza et al. (2014). Their analysis include observations from PIONIER 4 beam combiner, obtaining high precision visibility measurements with closure phase information. Its photosphere was studied in detail since no important circumstellar activity was detected from 2009 to 2011. The employed data include photometry, spectroscopy, polarimetry and interferometry (squared visibilities, closure and differential phases) all considered in a single analysis.

The adopted model for the stellar photosphere is the Roche model (rigid rotation and mass concentrated in the stellar center) with a generalized form of the von Zeipel's gravity darkening (RVZ model). The model-fitting was performed using the emcee code (Foreman-Mackey et al. 2013), an implementation of a Markov Chain Monte Carlo (MCMC) method. A summary of the Achenar's parameters is in Table 1.

The stellar diameter ratio $2 a / 2 b$ is about 1.352 , which corresponds to a not so high rotation rate. Still, it is the highest rotation rate observed in a angularly resolved star so far. The determined $\beta$ parameter supports the model of Espinosa Lara \& Rieutord (2011), which is a promising explanation to gravity darkening variation in rotating stars.

Independently of the parametric fitting, interferometric reconstruction image was performed using the MIRA software (Thiébaut 2008). The difference between the brightness distribution of the two methods is such that it excludes any circumstellar emission above $1.5 \%$ from the total flux in the considered epoch of the observations.

\section{The recent disk activity}

In the beginning of 2013 Achernar started a new Be active phase after about 7 years of quiescence, in which was mostly a normal B star. Our campaign aims to characterize the new formed disk using VLTI spectro-interferometry and its radiative transfer modeling using the HDUST code (Carciofi \& Bjorkman 2006, 2008). Specifically, we aim to characterize for the first time the mass loss rate $\dot{M}$ and the viscous diffusion coefficient $\alpha$ to the newly formed Be disk based on the VDD model (c.f. Rivinius et al. 2013b, and references therein). According to this model, the CS disk surface density $\Sigma(r)$ can be a function of time according to the Eq. 4.1. 


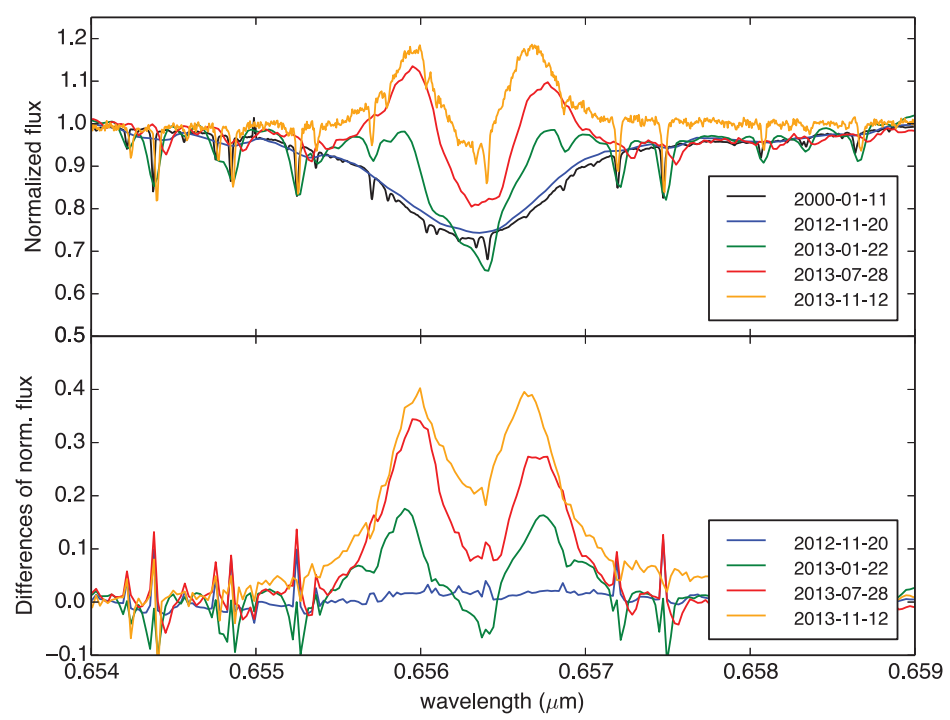

Figure 1. $\mathrm{H} \alpha$ line profile of Achernar.

$$
\frac{\partial \Sigma}{\partial t}=\frac{1}{\tau_{\text {vis }}}\left(\frac{2}{r} \frac{\partial}{\partial r}\left[r^{1 / 2} \frac{\partial}{\partial r}\left(r^{2} \Sigma\right)\right]+\Sigma_{\text {in }}(t) \frac{r_{\text {in }}^{2}}{r_{\text {in }}^{1 / 2}-1} \frac{1}{r} \delta\left(r-r_{\text {in }}\right)\right)
$$

The new activity has been report by Napoleão \& Marcon (priv. comm.) looking at the $\mathrm{H} \alpha$ line profile. The $\mathrm{H} \alpha$ evolution in 2013 can be seen in Fig. 1. As an example of phenomenon associated with the stellar activity is the Achernar's spin-up discovered by Rivinius et al. (2013a). So, this is a rare opportunity to evaluate the process of the evolution of the just formed Be disk high-angular resolution measurements: its importance is to unveil the spin unknown mass-loss mechanism(s) in Be stars and the process governing the circumstellar material in the close vicinity of the stellar photosphere in such a nearby star.

\section{References}

Carciofi, A. C. \& Bjorkman, J. E. 2006, ApJ 639, 1081

Carciofi, A. C. \& Bjorkman, J. E. 2008, ApJ 684, 1374

Carciofi, A. C., Domiciano de Souza, A., Magalhães, A. M., Bjorkman, J. E., \& Vakili, F. 2008, ApJ (Letters) 676, L41

Carciofi, A. C., Magalhães, A. M., Leister, N. V., Bjorkman, J. E., \& Levenhagen, R. S. 2007, ApJ (Letters) 671, L49

Domiciano de Souza, A., Hadjara, M., Vakili, F., et al. 2012a, A\&3A 545, A130

Domiciano de Souza, A., Kervella, P., Faes, D. M., et al. 2014, A\&A accepted

Domiciano de Souza, A., Kervella, P., Jankov, S., et al. 2003, AESA 407, L47

Domiciano de Souza, A., Vakili, F., Jankov, S., Janot-Pacheco, E., \& Abe, L. 2002, A\&A 393, 345

Domiciano de Souza, A., Zorec, J., \& Vakili, F. 2012b, in S. Boissier, P. de Laverny, N. Nardetto, R. Samadi, D. Valls-Gabaud, \& H. Wozniak (eds.), SF2A-2012: Proceedings of the Annual meeting of the French Society of Astronomy and Astrophysics, pp 321-324

Espinosa Lara, F. \& Rieutord, M. 2011, A\&A 533, A43

Foreman-Mackey, D., Hogg, D. W., Lang, D., \& Goodman, J. 2013, PASP 125, 306

Kanaan, S., Meilland, A., Stee, P., et al. 2008, A\&A 486, 785 
Kervella, P. \& Domiciano de Souza, A. 2006, A\& A 453, 1059

Rivinius, T., Baade, D., Townsend, R. H. D., Carciofi, A. C., \& Štefl, S. 2013a, A $\& A$ 559, L4

Rivinius, T., Carciofi, A. C., \& Martayan, C. 2013b, A\&A Rev. 21, 69

Thiébaut, E. 2008, MIRA: an effective imaging algorithm for optical interferometry

van Belle, G. T. 2012, A\&A Rev. 20, 51

Vinicius, M. M. F., Zorec, J., Leister, N. V., \& Levenhagen, R. S. 2006, A\& A 446, 643

\section{Discussion}

Rivinius: Can you comment on the presence (or absence) of a polar wind above Achernar?

FAEs: We have no reason to believe this feature was present in Achernar at the time of our observations. As we have shown in Domiciano de Souza et al. (2014) about the non-presence of a residual disk in the data, the hydrogen emission of such wind would be detected in linear polarization and spectroscopic line profiles before having an interferometric significance on IR.

STEE: There was a clear detection of a polar wind by Kervella \& Domiciano (2006) confirmed by Kanaan (2008) of about $5 \%$ in the $K$ band. Do you have any evidence of such wind in your data? If you add a $5 \%$ extended contribution along the polar axis, is it still compatible with your data?

FAEs: As said, there is no evidence of such feature. We have even tried to minimize the photospheric parameters of Achernar in addition with another Gaussian emission, having it free position, size and intensity. An additional component was unable to improve data fitting, comprising the characteristics of such polar axis emission. Furthermore, Achernar's reconstructed image differs from the data in a maximum of $1.5 \%$ in flux, and this difference is not extensive in the polar direction.

Peters: Have you attempted to incorporate the observed FUV photometric variability into your models? On the 1980-90's a 30\% flux variability with a period of $\sim 0.7$ day was observed in the FUV by instrumentation on Galileo [satellite]. A lesser variability at the same period was seen in IUE flux data. It seems to me that $\alpha$ Eri had a hot spot that rotated into/out of our line-of-sight.

FAEs: This is something that we have not done yet that is to compare its historical measured variability and our modeling. For example, Carciofi et al. (2007) detected linear polarization variations on timescales as short as $1 \mathrm{hr}$ and as long as several weeks. Their modeling strongly suggested that short-term variations can originate from discrete mass ejection events occurring at the photosphere-disk interface. To analyze the evolution of Achernar in light of the new photospheric parameters is the next step of our research. Thank you to remind us about theses UV records.

Domiciano De Souza: UV variations of 0.7 day period should be compared to the new photospheric model parameters. There is no indication of polar wind in the PIONIER data, but there is a residual flux of $1 \%$. The VINCI data show a decrease of visibility at low spatial frequency. This is an observational result. Other interpretations different from the polar wind can be tested in combination with the new data.

PULS: Just one comment regarding the "polar wind", that has been mentioned now a couple of times. Let me remind you that we have already discussed this in Paris (IAUS "Active OB-Stars"), and that a typical wind from a B-type would have a very low mass- 
loss rate $\left(\sim 10^{-8} M_{\odot} \mathrm{yr}^{-1}\right)$. The corresponding IR-emission would be very low, with $\tau \approx 1$ very close to the photosphere. So, what has been observed (whatever it is) is completely inconsistent with wind theory, whether prolate or not.

FAEs: I completely agree. As a suggestion, we could refer to this feature as interferometric polar signal instead of "polar wind" since there is no reason to relate this signal to a wind from the theoretical point of view.

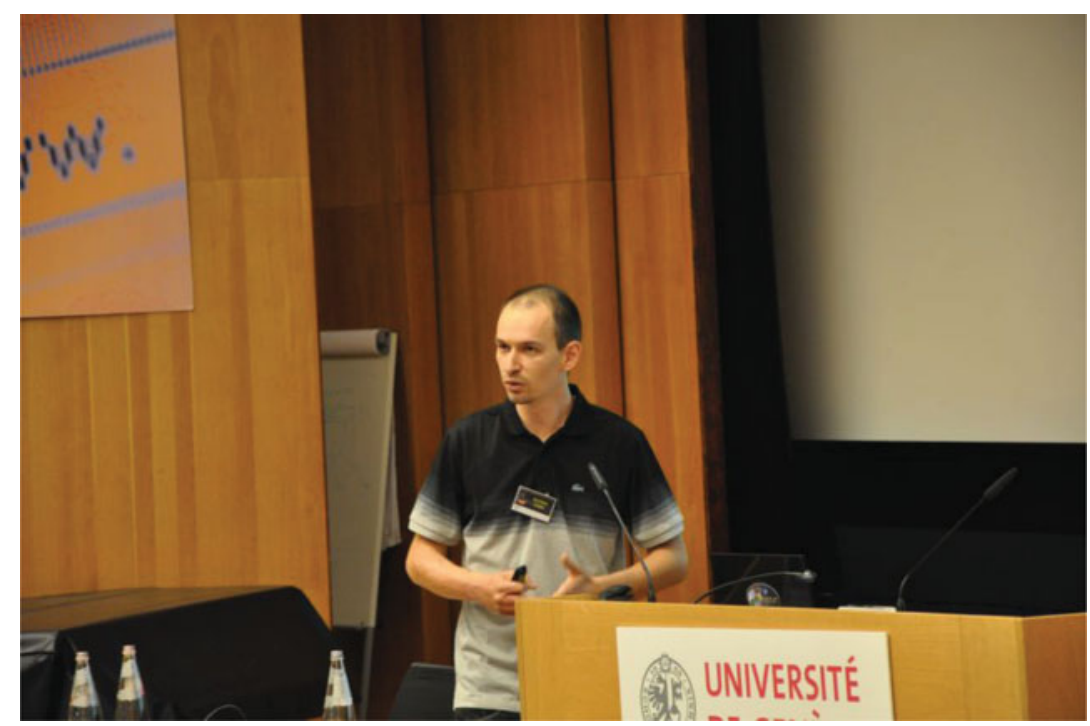

Daniel Faes

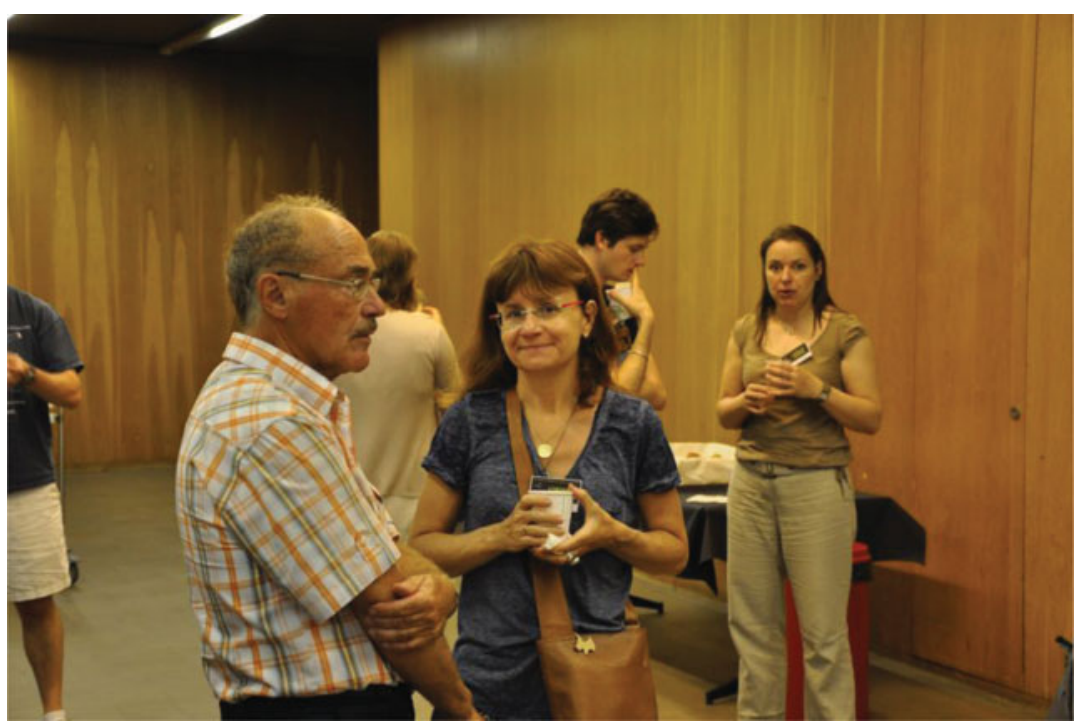

André Maeder and Corinne Charbonnel 